\title{
Chronic focal sclerosing osteomyelitis accompanied with bony mass protrusion into the maxillary sinus: a case report
}

\author{
Aya Yoshino $^{1}$, Seiji Kondo ${ }^{2}$, Masaaki Karino ${ }^{1}$, Yoshiki Nariai ${ }^{1}$, Michael Vieth ${ }^{3}$, Katsumi Hideshima ${ }^{1}$, \\ Hiroaki Ishibashi ${ }^{1}$, Joji Sekine ${ }^{1}$
}

${ }^{1}$ Department of Oral and Maxillofacial Surgery, Shimane University Faculty of Medicine, Izumo, Japan

${ }^{2}$ Department of Oral and Maxillofacial Surgery, Showa University School of Dentistry, Tokyo, Japan

${ }^{3}$ Institut für Pathologie, Klinikum Bayreuth GmbH, Bayreuth, Germany

Abstract: This paper describes the first documented case of chronic focal sclerosing osteomyelitis accompanied by bony mass protrusion into the maxillary sinus manifested in a 34-year-old Japanese woman. The lesion was a circumscript radiopaque mass in the left maxillary sinus close to the apex of the left upper second molar. The tooth and adjacent molars were vital, and there was no apparent inflammation, though diffuse and sclerotic changes were observed around the left molar region of the maxilla on simple X-ray and CT images. Histopathological examinations of resected tissue showed that the protrusive hard mass was composed of dense bony trabeculae with prominent mature lamella covered by mucosal epithelia of the maxillary sinus with an inner granulation tissue zone, and of fibrous granulation tissue fragments with focal dense inflammatory cells including foamy macrophages and plasma cells and prominent Russell body formations. The patient was finally diagnosed as having chronic focal sclerosing osteomyelitis based on the radiographic and clinicopathological findings. As of about 35 months after the operation, there has been no sign of recurrence.

[Oral Med Pathol 2011; 15: 91-94 doi: 10.3353/omp.15.91]

Key words: chronic focal sclerosing osteomyelitis, maxillary sinus, bony mass protrusion, odontogenic infection, pulp necrosis

Correspondence: Joji Sekine, Department of Oral and Maxillofacial Surgery, Shimane University Faculty of Medicine, 89-1 Enya-cho, Izumo 693-8501, Japan.

Phone: +81-853-20-2301, Fax: +81-853-20-2299, E-mail: georges@med.shimane-u.ac.jp

\section{Introduction}

Chronic focal sclerosing osteomyelitis (CFSO) is a lesion in which sclerotic bone is formed at the apex of a non-vital tooth as a result of a low-grade infection of the dental pulp (1). Eliasson et al. reported a female-to-male incidence ratio of 3 to 2 , and $50 \%$ of the cases were in patients under 30 years of age (2). Pain is characteristically mild or absent, and there is no swelling, lymphadenopathy, or jaw expansion (1). Traumatic malocclusion may play a role in the pathogenesis of CFSO (3). Eighty-five percent of CFSO cases occur mainly in the mandible, especially in the lower first molar region (4). CFSO rarely occurs in the maxilla, probably because of the thin cortical bone and rich collateral blood supply in the maxilla (5), and it often remains undetected unless incidentally found on a routine radiographic survey (6).

This paper describes an extremely rare case of CFSO accompanied by a bony mass protrusion into the maxillary sinus, and discusses the clinical management and etiology of CFSO.

\section{Case Report}

A 34-year-old Japanese woman was referred to the Department of Oral and Maxillofacial Surgery, Shimane University Hospital by her dental practitioner for extra examination of the left maxillary sinus. A radiopaque mass lesion was pointed out in the posterior wall of the left maxillary sinus by orthopantomogram.

At the time of the referral, the patient had no symptom in any teeth. Intraoral examination did not reveal any obvious decayed teeth. Extraoralexamination did not show any swelling or tenderness in the oral cavity. Orthopantomogram showed a radiopaque mass lesion with a clear margin close to the apex of the left upper second molar in the posterior wall of the left maxillary sinus. In addition, there were diffuse and sclerotic bony changes in the bone marrow zone in the left upper molar region, especially around the second molar (Fig. 1). Computed tomographic (CT) scans showed a high-density mass measuring about $17 \times 15 \times 13 \mathrm{~mm}$ accompanied by an internal low-density area in the left maxillary sinus floor and close to the apex of the left upper 


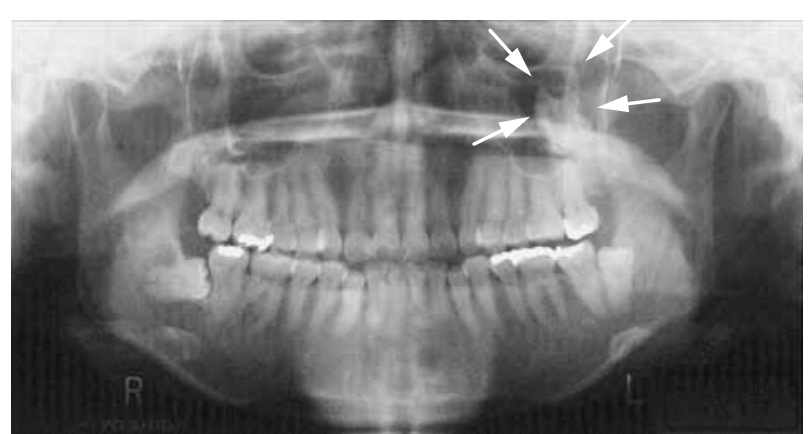

Fig. 1. Preoperative orthopantomogram of chronic focal sclerosing osteomyelitis (CFSO) arising in the left maxillary molar region of a 34-year-old Japanese woman. A focal radiopaque mass in the left maxillary sinus floor and above the apex of the left upper second molar (arrows) was seen with diffuse osteosclerotic changes around the molar teeth.

second molar (Figs. 2a, b). Sclerotic change was also confirmed in the surrounding bone marrow of left upper second molar (Fig. 2b). The mean CT value of the highdensity mass was $967 \mathrm{HU}$; the lesion appeared as a radiopaque mass with a density similar to normal bone. Meanwhile, an internal low-density area was valued at 53 HU, similar to soft tissue (Fig. 2a). In an extra examination of the left upper second molar, a cast metal inlay was applied. Electric pulp test showed a slightly weak response compared with the right upper second molar; the percussion test was negative. The patient's past medical history was unremarkable. The clinical diagnosis was suspicion of odontogenic tumor, such as calcifying cystic odontogenic tumor, periapical cemental dysplasia, or osteoma, in the left maxillary sinus.

Tumorectomy was then performed under general anesthesia. A straight line incision in the left upper vestibule was made to gain access to the left lateral maxillary wall, and the mucoperiosteal flap was elevated to expose the sinus wall. A bone window was created with a small surgical saw, with the mucous membrane carefully left intact. The mucous membrane of the maxillary sinus was then incised, and the lesion was resected with a surgical margin without exposing the apex of the left upper second molar (Fig. 3). The bone window was then replaced and secured by closing the oral mucosal flap with absorbable surgical sutures.

Although the postoperative course was uneventful, no response was seen by the electric pulp test about 7 months postoperatively. Root canal treatment of the affected tooth was then provided by the patient's family dentist. Recurrence or inflammatory findings in the left maxillary sinus were not seen in CT and orthopantomogram images 35 months later.

\section{Pathological findings}

Macroscopically, the excised specimens were composed of one major hard tissue mass covered by the sinus mucosa, measuring about $17 \times 15 \times 13 \mathrm{~mm}$ in diameter. The covering mucosa was grossly blanched, indicating mild inflammation. In addition to the hard tissue mass, scattered granulation tissue containing hard tissue fragments were obtained at surgery (Fig. 4a). Histologically, the main hard tissue consisted of cortical bone tissue with irregularly thickened bone trabeculae which had complicated but mature lamellation. The inner part of the protrusive mass lacked bony trabeculae and was filled with fibrous granulation tissue (Fig. 4b). The surface of the bony tissue was covered by mildly thickened sinus mucosa undergoing fibrosis (Fig. 4c). The bone marrow or the interbony-trabecular space was filled with fibrous granulation tissue without fatty marrows, as well as with thick and irregular-shaped bone trabeculae (Fig. 4c). In high power view, there were various fibrotic areas with a trace of fatty marrow (Fig. 4d). The small fragments of granulation tissue were in various stages of inflammation, from immature to fibrotic ones with newlyformed bone trabeculae, which might have been from the inner radiolucent part of the lesion, as confirmed in Fig. 4b. There were foci of immature granulation tissue with dense infiltrations of chronic inflammatory cells within the small fragments as seen in Fig. 4a (Fig. 4e). Most of the inflammatory cells were plasma cells and foamy macrophages. There were Russell bodies over the dense aggregates of plasma cells (Fig. 4f), which is a common histological feature of periapical granuloma or radicular cyst, indicating that the lesion was still exposed to inflammatory stimuli. Based on the above-mentioned findings, a diagnosis of CFSO possibly due to molar tooth infection was made.

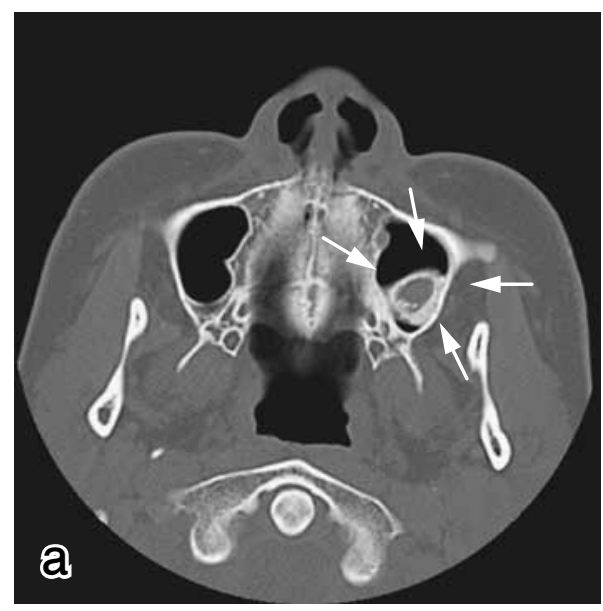

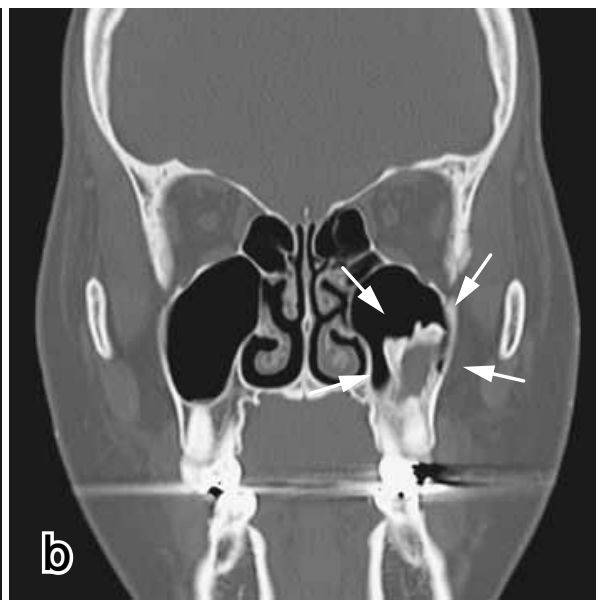

Fig. 2. Preoperative CT scan images of CFSO. (a) Axial section of bone density; (b) Coronal section of bone density. A high-density mass lesion, measuring about $15 \times$ $13 \times 17 \mathrm{~mm}$ in size, with an internal low-density area in the left maxillary sinus floor and close to the apex of the left upper second molar was shown (arrows). Mean CT values were $967 \mathrm{HU}$ for the peripheral part, and $53 \mathrm{HU}$ for the internal area $(a, b)$. 


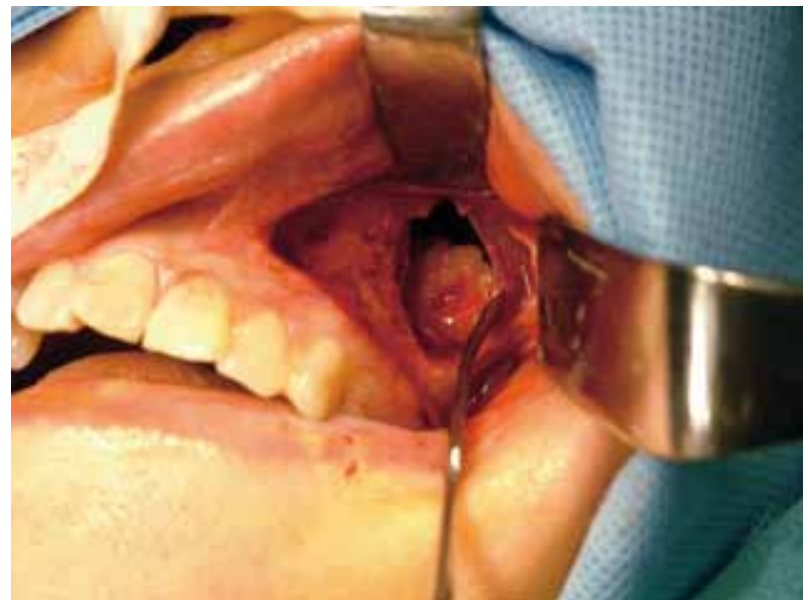

Fig. 3. Intraoperative finding of an intrasinus mass due to CFSO. After a bone window was created on the lateral wall of the left maxillary sinus, the lesions were extracted and the piece of bone was retained, repositioned, and stabilized with sutures to its original position.

\section{Discussion}

Initially, a diagnosis of CFSO could not be established because no clinical sign of inflammation was seen, and because CFSO has been reported to be most common in the mandible accompanied with non-vital teeth (6). In our case, as excised lesion was a hard tissue mass, odontogenic tumors, such as odontoma and osteoma, should be distinguished.

Histopathologically, the excised hard tissue consisted of sclerotic bone with prominent mature lamella, and the bone marrow was replaced with fibrous tissue and infiltrated by inflammatory cells without odontogenic tissue. Concerning the histopathologic variants of osteoma, one was composed of relatively dense compact bone with sparse marrow tissue, the other of lamellar trabeculae of cancellous bone with fibrofatty marrow spaces (7). Neither odontogenic tumor nor osteoma could be denied considering the CT image showing a low-density area in the hard tissue mass. On the other hand, the soft tissue taken from the surroundings of the apex of the diseased tooth or from the inner part of the protrusion consisted mainly of mature collagenous tissue with mild infiltration of chronic inflammatory cells. Those histopathological findings seemed to be the reaction against mild chronic inflammation which had infiltrated from the tooth and/or maxillary sinus base.

The pathogenesis of CFSO has been reported as resulting from mild and continuous infection of the pulp (1, $5)$. It is usually seen at the apex of a tooth in which there has been a long-standing pulpitis (7). In our case, preoperatively, though the left maxillary sinus showed clear features, the electric pulp test of the left upper second molar revealed a slightly weak response. In the operation, the lesion was completely resected without exposing the apex of the left upper second molar, and then the bone window was replaced (8). Though the postoperative course, including that of the maxillary sinus, was uneventful, the electric pulp test of the left upper second molar showed the tooth to be completely necrosed about 7 months postoperatively.

Regarding the etiology of our case, chronic low-grade
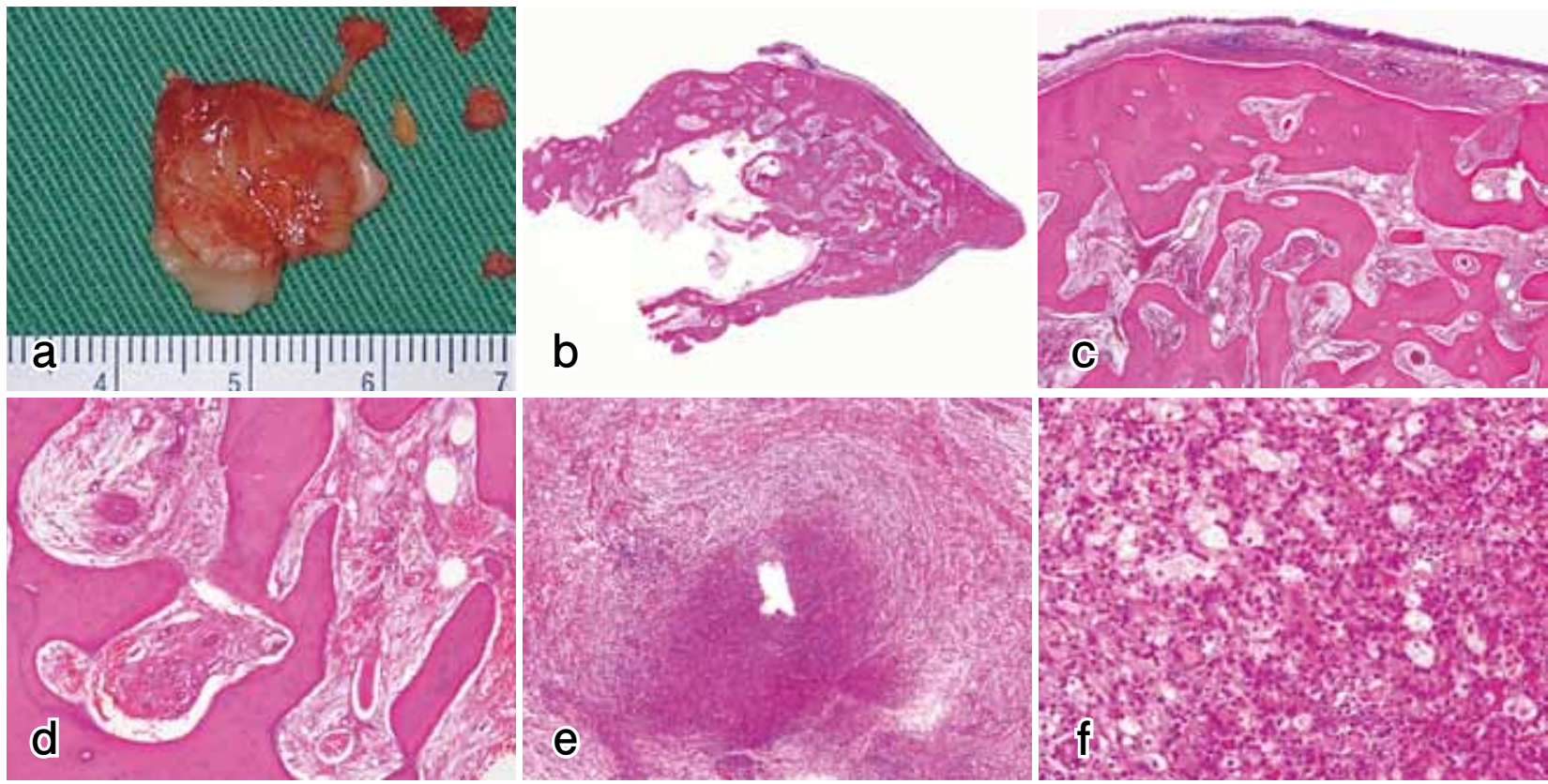

Fig. 4. Surgical specimens of CFSO. (a) Macroscopic view of the excised specimens. (b-f) Histology of removed tissue specimens, hematoxylin and eosin stain. (b) Main hard mass; $\times 12$, (c) main hard mass, $\times 70$; (d) high power view of panel (c) $\times 200$; (e) small fragments of granulation tissue, $\times 100$; (f) high power view of panel $(\mathrm{e}), \times 400$. The main hard tissue was a fragment of cortical bone covered by the sinus mucosa. Its inner part lacked bony trabeculae and was filled with fibrous granulation tissue (b). Its surface was covered by the maxillary sinus mucosa with chronic inflammation (c). The marrow space was replaced with fibrous granulation tissue with thick and regular-shaped bony trabeculae with complicated lamellation. There was a trace of fatty marrow (d). There were foci of immature granulation tissue with dense infiltrations of chronic inflammatory cells within the small fragments seen in panel $(a, e)$. The major inflammatory cells were plasma cells and foamy macrophages. There were Russell bodies over the dense aggregates of plasma cells (f). 
inflammation around the apex of the left upper second molar by partial pulp necrosis was then thought to induce reactive osteogenesis, which manifested as a focal radiopaque mass into the maxillary sinus.

In conclusion, although CFSO is an uncommon disease in the maxillary sinus, it should be included in the differential diagnosis of amorphous radiopaque masses close to dental roots in the maxillary sinus.

\section{References}

1. Douglass GD, Trowbridge HO. Chronic focal sclerosing osteomyelitis associated with a cracked tooth: Report of a case. Oral Surg Oral Med Oral Pathol 1993; 76: 351-5.

2. Eliasson S, Halvarsson C, Ljungheimer C. Periapical condensing osteitis and endodontic treatment. Oral Surg Oral Med Oral Pathol 1984; 57: 195-9.

3. Eversole LR, Stone CE, Strub D. Focal sclerosing osteomyelitis/focal periapical osteopetrosis: radiographic patterns. Oral Surg Oral Med Oral Pathol 1984; 58: 45660.

4. Farman AG, De V Joubert JJ, Nortje CJ.Focal osteosclerosis and apical periodontal pathosis in "European" and Cape coloured dental out patients. Int J Oral Surg 1978; 7: 54957.

5. Barry CP, Ryan CD. Osteomyelitis of the maxilla secondary to osteopetrosis: Report of a case. Oral Surg Oral Med Oral Pathol 2003; 95: 12-5.

6. Cawson RA, Binnie WH, Barrett AW, Wright JM. Oral Disease, 3rd edition. CV Mosby, St. Louis, 2001; 9-10.

7. Joseph AR, James S. Oral Pathology Clinical-Pathologic Correlations, 2nd edition. WB Saunders, Philadelphia, 1993; 379-435.

8. Lundgren S, Andersson S, Gualini F, Sennerby L. Bone Reformation with Sinus Membrane Elevation: A new surgical technique for maxillary sinus floor augmentation. Clin Implant Dent Relat Res 2004; 6: 165-73. 